\title{
Severe Constipation in a Wheelchair-Bound, Elderly Woman
}

\author{
Shrikant K. Tamhane, DO'; Jace Tamhane, DO² \\ ${ }^{1}$ Southbay Family Medicine Clinic, Carson, CA \\ ${ }^{2}$ St. Barnabas Emergency Medicine Residency Program, Bronx, NY
}

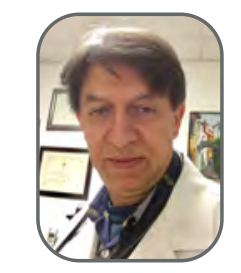

Shrikant K. Tamhane, DO

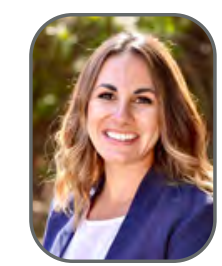

Jace Tamhane, DO

KEYWORDS:

Constipation, Osteopathic Manipulative Medicine

\section{ABSTRACT}

Constipation is a common household word. Effective remedies in most cases are also simple household remedies such as over-the-counter medicines, lifestyle modification, and a high fiber diet. In the hospital setting, constipation is multifactorial, with multiple etiologies explained in this case study. Complications can lead to abdominal pain and more serious complications like ischemic bowel, toxic megacolon, perforation, and even death. Causes of serious complications include diabetic gastroparesis, paralytic ileus, or surgical adhesions. These are explored in the paper and gives the reader a deeper realistic medical insight into the seriousness of the situation. Osteopathic techniques to treat constipation are discussed and demonstrated to the caregiver with great response.

\section{INTRODUCTION}

Chronic constipation is a complex and multifactorial intestinal disorder thought to be idiopathic in nature or secondary to many disease processes and medications. It is the most common presenting digestive complaint with a prevalence estimated between 12 and 19 percent, more common in women, and increases with age. The most dramatic rise is at age 65, with approximately 26 percent of men and 34 percent of women experiencing constipation. Other risk factors include physical inactivity, low education and income, concurrent medication use, and depression. ${ }^{1}$ There are currently two schools of thought when it comes to constipation. The American Gastroenterological Association uses colonic transit and anorectal tests to classify constipation into one of the three groups: normal transit constipation, slow-transit constipation, and pelvic floor dysfunction or defecatory disorders. In contrast, pharmaceutical trials and epidemiologic studies use the Rome IV criteria, which defines constipation as meeting two or more of the following: stool frequency less than three per week, straining during defecation, having hard or lumpy stool, a sensation of incomplete evacuation or anorectal blockage, and the use of manual maneuvers to facilitate bowel movement. Using one classification system versus the other depends on clinician familiarity. Regardless, clinicians should be aware of the current guidelines and systematic 
approach to treating constipation and rule out any secondary causes of constipation. This can become a chronic and debilitating disease for patients and lead to a significant economic burden.

\section{CASE PRESENTATION}

A 65-year-old African American female presents with five months of ongoing constipation, stating she has a bowel movement "every other week." She currently uses a wheelchair due to a CVA that occurred in March 2018, resulting in L-sided hemiparesis. Her past medical history is significant for type II diabetes mellitus (DM) with diabetic neuropathy, hypertension, and degenerative joint disease. Patient initially presented on 8/11/2020 with nausea, vomiting, and decreased appetite, all thought to be secondary to Metformin medication. In addition, she complained of having 1-2 bowel movements (BM) per week. We recommended increasing fiber and fluid intake, prescribed a stool softener and stimulant laxative. At the one-week follow-up, patient admitted to persistent nausea and vomiting along with two days of diarrhea, proceeded by no BM for five days. At this time, an osmotic agent and an anti-emetic agent were prescribed. Patient returned on 10/9/2020 stating, "Miralax package didn't work at all." Upon physical exam, she had hypoactive bowel sounds in all four quadrants. Again, a stimulant laxative, Dulcolax, was prescribed. She returned on 1/15/2021 with severe constipation, stating she has a BM "every other week." She denied nausea, vomiting, diarrhea, and abdominal pain. Upon physical exam, bowel sounds were hyperactive in all four quadrants. Patient tried a suppository without relief. She was given a fleet enema and soap enema. Osteopathic manipulation therapy (OMT) was recommended at this time. We taught the caregiver bowel mobilization techniques to be completed two times a day $x$ seven days. They returned on 1/22/21, stating the patient has had a BM every day for the past seven days. They admitted to doing the OMT techniques along with giving a tablespoon of olive oil every morning.

\section{DISCUSSION}

Of the many etiologies that can bring about constipation, we will address the causes associated with this patient presentation. She had many risk factors, including gender, age, low socioeconomic status, and an inactive lifestyle secondary to using a wheelchair. Her past medical history was pertinent for diabetes mellitus and cerebrovascular accident (CVA) both known for perpetuating constipation. Gastroparesis, delayed gastric emptying, is a welldocumented disease highly associated with type 1 and type II DM, and those with this complication have worse outcomes and morbidity. ${ }^{3}$

Normal gastrointestinal motor functions are coordinated by a complex interplay between enteric, sympathetic, and parasympathetic nerves communicating between the brain, stomach, and intestines. Digestion begins as the food bolus is transmitted via peristaltic propulsions from the esophagus to the stomach. Mechanosensitive receptors are activated via the Vagus nerve to allow the stomach to expand, creating a reservoir for the food, called accommodation. Special enteric nervous system cells called interstitial cells of Cajal are activated, propelling the food bolus forward and creating rhythmic contractions grinding food into small pieces until they are ready to be emptied. The antroduodenal reflex allows appropriately sized particles to be released into the duodenum, where they can be absorbed into our bodies. Diabetes has been shown to affect multiple levels in this process, including postprandial accommodation and reduced frequency of antral contractions. These are thought to result from impaired autonomic dysfunction or abnormalities in the enteric nervous system, such as the interstitial cells of Cajal.

Another contributing factor to consider for our patient was the CVA resulting in $L$ sided hemiparesis. Studies have shown that constipation is more prevalent in neurologic disorders, especially cerebral ischemia. Incidences of constipation after an ischemic stroke have been reported as high as 55\% four weeks after and 30\% after three months. ${ }^{2}$ The exact pathophysiology remains poorly understood, but it is currently postulated that cerebral ischemia may damage various cortical and medullary nuclei leading to interruption of the brain-gut axis. This alters the neural circuits controlling essential GI functions such as sphincter function and normal peristalsis. At a more fundamental level, the CVA left our patient in a wheelchair, leading to her immobile and sedentary lifestyle. The current thought is that movement of our body and gravity both assist in the movement of stool through the GI tract. 
Treating chronic constipation in an evidence-based, systematic way is of value to the clinician as this will reduce complications associated with the chronicity of this disease. We worry about hemorrhoids and anal fissures early on due to prolonged straining with increased intra-abdominal pressure and hard stool passing through the rectum during evacuation. As constipation progresses, fecal matter can build up within the colon leading to fecalith impaction and eventually obstruction. Prolonged stasis can compress the walls of the colon resulting in an ischemic ulcer. There is a risk of this ulcer rupturing and perforation of fecal matter into the abdomen as with any ulcer.

\section{TREATMENT}

Each treatment should be tailored towards the individual's needs and what comorbid conditions are present, making sure to rule out all secondary causes of constipation. Initial therapy should always be a lifestyle and dietary modification, including recommendations on increasing water intake, increasing physical activity, and eating more foods rich in fiber. The first line of medication therapy to consider are bulk laxatives, with psyllium being shown to be the most efficacious in three randomized control trials (RCTs). Bulk laxatives work by pulling water into the bowel lumen and enhancing bowel movements. It is necessary to inform patients to consume adequate water, or it only worsen symptoms. The second-line medication to consider is osmotic laxatives, with polyethylene glycol (PEG) being far superior. PEG and other osmotic laxatives are hyperosmolar, thereby retaining water in the colon, enhancing stool passage. Osmotic laxatives can be supplemented with stimulant laxatives, senna, and bisacodyl when necessary. ${ }^{4}$

It is important to note that stimulant laxatives are efficacious, but chronic use should be avoided due to the unknown long-term effects. If these two treatment trials have failed, newer agents are available called secretagogues with Lubiprostone shown to be most efficacious. Lubiprostone is a chloride channel activator that actively secrete chloride with water following into the intestinal lumen. Stool softeners such as docusate used to be another first-line agent to consider, but there is limited evidence to support its efficacy, so recommendation is low. Enemas should be used to prevent fecal impaction in patients with several days of impaction.

As osteopathic physicians, we must consider osteopathic manipulation to aid in the treatment of our patients. Limited clinical trials are studying OMT on chronic constipation, but few trials have shown that OMT is efficacious in treating constipation. The treatment modalities that can be utilized are rib raising, suboccipital release, lumbosacral decompression, and lumbar paraspinal inhibition, which help normalize autonomic tone. A more direct visceral technique of the gut can be performed to free restrictions of the viscera and help mobilize the colon, focusing first on the splenic and hepatic flexures, then moving to the cecum and sigmoid colon. Finally, a colonic milking technique can be applied to start at the ascending colon, working proximal to the distal, to the transverse, and down to the descending colon, mimicking the natural movement of stool through the colon. During our encounter with the patient, I performed paraspinal inhibition and the colonic milking technique, which each take about 1-2 minutes to complete. I then was able to teach her caregiver these simple techniques to be performed at home.

A fee based step-by-step demonstration course of these OMT techniques (among 150 available) can be found at https://www.pathlms.com/acofp/courses/13565.

\section{DIFFERENTIAL DIAGNOSIS}

1. Chronic constipation secondary to diabetic gastroparesis 2. Paralytic ileus secondary to stroke

\section{AUTHOR DISCLOSURES:}

No relevant financial affiliations or conflicts of interest.

\section{REFERENCES:}

1. Adil E. Bharucha, Arnold Wald. Chronic Constipation. Mayo Clinic Proceedings, Volume 94, Issue 11, 2019, Pages 2340-2357. ISSN 0025-6196.

2. Carlos R. Camara-Lemarroy, Beatriz E. Ibarra-Yruegas, Fernando Gongora-Rivera. Gastrointestinal complications after ischemic stroke. Journal of the Neurological Sciences, Volume 346, Issues 1-2, 2014, Pages 20-25. ISSN 0022-510X.

3. Christophe Vanormelingen, Jan Tack, and Christopher N. Andrews. Diabetic Gastroparesis. British Medical Bulletin 2013; 105: 213-230. DOI:10.1093/bmb/ldt003

4. Lawrence Leung, Taylor Riutta, Jyoti Kotecha, Walter Rosser. Chronic Constipation: An Evidence-Based Review. The Journal of the American Board of Family Medicine Jul 2011, 24 (4) 436-451; DOI: 10.3122/jabfm.2011.04.100272 